\title{
Coupling and phase analysis of cavity structures in two-dimensional photonic crystals
}

\author{
Kaan Guven and Ekmel Ozbay \\ Department of Physics, Bilkent University, Bilkent 06800, Ankara, Turkey \\ and Advanced Research Laboratory, Bilkent University, Bilkent 06800, Ankara, Turkey
}

(Received 8 June 2004; revised manuscript received 16 August 2004; published 9 February 2005)

\begin{abstract}
An experimental and numerical study of the coupling properties of cavity waveguides and the phase advancement through the coupled cavity waveguides in two-dimensional photonic crystals is presented. We find that the resonance frequency is insensitive to the confinement strength for cavity and for finite size defects. We obtain the coupling constant as a function of the distance between two cavities within the tight-binding formalism experimentally, and show the exponential decay of the coupling strength. By measuring the phase spectra of coupled cavity waveguides (CCW's), we clearly demonstrate that the phase shift across the guiding band is equal to $N \pi$, where $N$ is the number of cavities in the waveguide. This essential behavior is employed to analyze the transmission response of CCW-based Mach-Zehnder interferometer structures introduced recently.
\end{abstract}

DOI: 10.1103/PhysRevB.71.085108

PACS number(s): 42.70.Qs, 42.60.Da, 42.25.Hz

\section{INTRODUCTION}

Waveguide structures are essential components of photonic integrated circuits. In photonic crystals (PC's), it is proposed $^{1}$ and shown ${ }^{2-6}$ that the electromagnetic wave can be guided through sharp bends and intersections with high transmission characteristics. Various designs for waveguide bends have been studied to improve the transmission. ${ }^{7-10}$ Following the proposal by Yariv et al.,$^{11}$ coupled cavity waveguides (CCW's) in photonic crystals have become an intense research subject in recent years, as they provide some advantages over conventional PC-based waveguides. ${ }^{11-14}$ These advancements open a way to construct extremely compact and efficient integrated photonic circuits. ${ }^{5,6,15,16} \mathrm{Nu}-$ merous CCW-based fundamental optical device components (e.g., splitters, switches, channel add-drop filters) have been demonstrated in the microwave regime and are being readied for the optical regime. In particular, CCW's can be utilized to construct ultracompact optical delay lines which has a direct impact on the overall device size. ${ }^{17}$ The application potential of CCW's drives in turn the investigation of basic optical properties in detail, such as the coupling properties of cavities. Lan et al. ${ }^{18}$ pointed out this necessity and conducted a systematic analysis of defect coupling in one- and twodimensional photonic crystals using transfer matrix formalism.

In the first part of the paper, we aim to extend the investigation of the coupling properties of CCW's experimentally and numerically. In particular, we show the exponential decay of localized modes within the cavities, by measuring the coupling strength of two cavities as a function of the intercavity distance. For this purpose, we employ the tightbinding formalism. Surprisingly, we find that even the resulting eigenmodes of two merged cavities obey the tightbinding formalism very well, despite the anticipated strong interaction between the individual cavity modes.

In the second part of the paper, we investigate the phase delay spectra of single and coupled cavity structures. It is well known that, in addition to improved transmission characteristics, the slow group velocity at the guiding band edges promotes CCW's as promising candidates for optical delay lines. An example is the CCW-based Mach-Zehnder interferometer (MZI), proposed and demonstrated recently. Soljačić et al. investigated the phase shift induced by nonlinear effects in CCW-based MZI numerically. ${ }^{19}$ Later, Martinez et $a l .{ }^{20}$ demonstrated experimentally a MZI structure, with a simple transfer function model utilizing the difference in the number of cavities of the respective interferometer branches. Both studies emphasize the compact size of the structures that is required to attain the operational phase shift of $\pi$. To this end, we measure the phase delay of CCW's, and demonstrate that the phase shift through a cavity is equal to $\pi$, which then extends linearly to $N \pi$ for a $\mathrm{CCW}$ consisting of $N$ cavities. This leads to a clear interpretation of the operation of a CCW-based MZI in terms of the phase difference (i.e., difference in the number of cavities) between the respective branches of the interferometer, and provides support to the transfer function model introduced before. ${ }^{20}$

This paper is organized as follows: In the second section, we describe the experimental setup and present the measurements of confinement effects on a single cavity and the coupling strength between two cavities as a function of the intercavity distance. The measurement of the phase spectra of single cavity and CCW structures is discussed in the third section. In the fourth section, we employ the results of the third section to analyze the operation of CCW-based MZI structures. We conclude by summarizing the results.

\section{COUPLED CAVITIES: CONFINEMENT EFFECTS AND THE COUPLING CONSTANT}

The photonic crystal in this study consists of a hexagonal array of cylindrical alumina rods with radius $r=1.575 \mathrm{~mm}$ and a refractive index of $n=3.13$ at microwave frequencies. The lattice constant is chosen as $a=7.0 \mathrm{~mm}$. The transmission and phase spectra are measured using a network analyzer and a set of horn antennas in TM polarization [electric field $E$ perpendicular to the plane of two-dimensional (2D) photonic crystal]. In all measurements, the major propaga- 


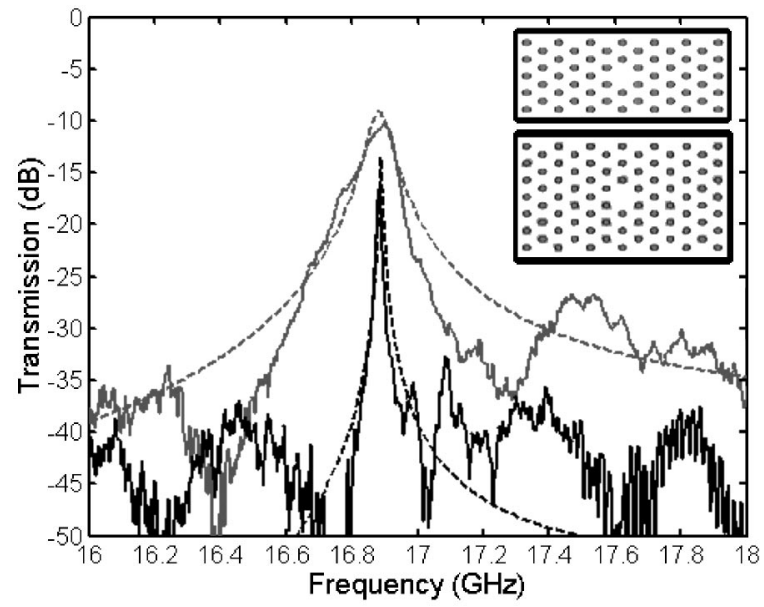

FIG. 1. Measured (solid lines) and simulated (dashed lines) transmission spectrum of a single cavity for the number 2 (gray) and 3 (black) of confining layers. Top right: Schematics of the respective structures (full lateral width is not shown).

tion direction is set along the $\Gamma K$ direction of the photonic crystal, unless otherwise noted. Numerical simulations based on the finite-difference time-domain (FDTD) method are used to compare with experimental data.

We first measured the transmission spectrum of the uniform photonic crystal, which exhibits a band gap between 12.8 and $18.7 \mathrm{GHz}$. A single cavity is formed by removing a rod within the photonic crystal, which appears as a localized mode within the gap of the transmission spectrum. For the investigation of the confinement, two PC structures are constructed having $5 \times 29$ and $7 \times 29$ layers, respectively. Since the crystal is much thicker along the lateral direction, the confinement depends on the number of layers around the cavity along the propagation direction, which we denote as "cap layers" in this paper. For the present structures, the number of cap layers are 2 and 3, respectively. Figure 1 shows the transmission spectra of a single cavity for two confinement strengths, which are depicted by the schematics in the figure. The cavity mode frequency located at $f$ $=16.898 \mathrm{GHz}$ appears to be independent of the confinement strength. This agrees well with the transfer matrix results where no significant dependence of resonant frequency on defect size is reported. ${ }^{18}$

It is evident that the spectral width and the transmission amplitude shrinks with increasing confinement, as expected. We further note that the dashed lines obtained by FDTD measurements are in very good agreement with the measurements.

Figure 2 shows the transmission spectra for two defects of radii 0.645 and $6.06 \mathrm{~mm}$, respectively. The larger defect exhibits two modes, from which only the high-frequency mode is shown on the figure. For the small defect, the resonance frequency is $f=15.98$ and $16.02 \mathrm{GHz}$ for strong and weak confinements, respectively. In the case of large defect, the resonance occurs at $f=16.90$ and $16.92 \mathrm{GHz}$, respectively. Even though a shift of resonance frequency is noticeable, we refrain from attributing this shift solely to the finite size of the defect. This is because the small distortions of the rod positions while modifying the confinement strength (i.e., re-

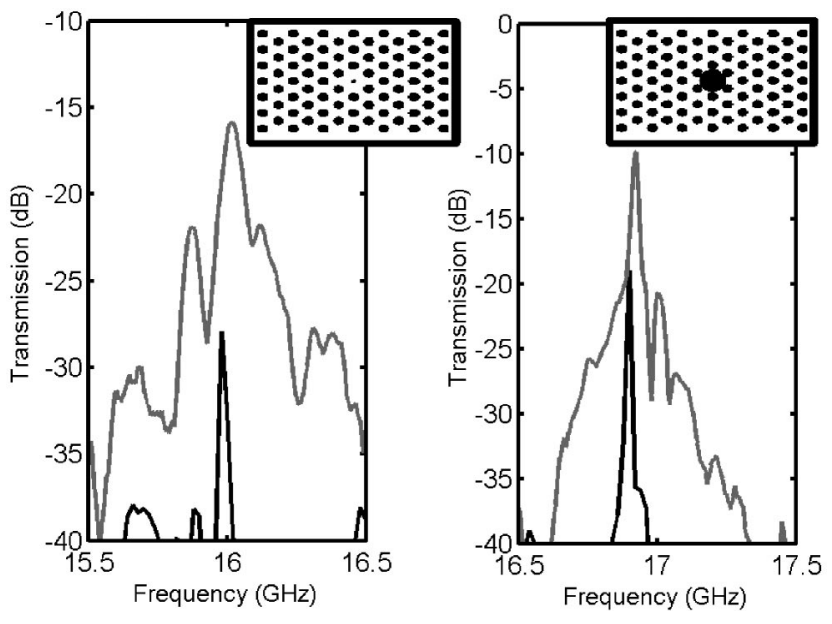

FIG. 2. Transmission spectra for a defect of radius $r$ $=0.645 \mathrm{~mm}$ (left panel) and of a defect of radius $r=6.06 \mathrm{~mm}$ (right panel). Upper (lower) curves correspond to weak (strong) confinement. Schematics display the strong confinement structures for respective defect sizes.

moving or adding rods to cap layers) may alter the frequency of the cavity and cause a shift of similar magnitude.

Although the confinement strength does not alter the resonance mode, the spectral broadening of the mode depends on it. We find that adding one more cap layer to each end of the crystal increases the confinement drastically, evident from the decrease of the spectral width of the mode.

If two or more cavities are present, it is well known that the eigenmode of the single cavity splits into coupled modes. This phenomenon is investigated experimentally and described within the classical wave analog of the tight-binding (TB) approximation for photonic crystal structures in detail. ${ }^{11-14,21-23}$ In the following, we will present the dependence of the coupling parameter to the intercavity distance for two cavities. Based on the formulation of Ref. 12, the eigenfrequencies of two coupled cavities are given by

$$
\omega_{1,2}=\Omega \sqrt{\frac{1 \pm \beta_{1}}{1 \pm \alpha_{1}}}
$$

where $\alpha_{1}$ and $\beta_{1}$ are the first-order coupling parameters defined as

$$
\begin{gathered}
\alpha_{1}=\int d \vec{r} \varepsilon(\vec{r}) \vec{E}_{\Omega}(\vec{r}) \cdot \vec{E}_{\Omega}(\vec{r}-\Lambda \hat{x}), \\
\beta_{1}=\int d \vec{r} \varepsilon_{0}(r-\Lambda \hat{x}) \vec{E}_{\Omega}(\vec{r}) \cdot \vec{E}_{\Omega}(\vec{r}-\Lambda \hat{x}) .
\end{gathered}
$$

Here, $\vec{E}_{\Omega}(\vec{r})$ describes the single-cavity mode, $\varepsilon_{0}(\vec{r})$ and $\varepsilon(\vec{r})=\varepsilon(\vec{r}-\Lambda \hat{x})$ are the dielectric functions of the single- and coupled-cavity systems, respectively, and $\Lambda$ is the distance between the cavities.

For an array of cavities, where each cavity interacts weakly with neighboring cavities, a defect band (waveguiding band) is formed. Similar to the two-coupled-cavity case, 
TABLE I. The resonant frequencies and corresponding tight-binding coupling constants of two coupled cavities for different intercavity distances, obtained by measurements and calculated from FDTD simulations.

\begin{tabular}{|c|c|c|c|c|c|c|}
\hline \multirow[b]{2}{*}{ Distance } & \multicolumn{3}{|c|}{ Experiment } & \multicolumn{3}{|c|}{ Simulation } \\
\hline & $f_{1}(\mathrm{GHz})$ & $f_{2}(\mathrm{GHz})$ & $\kappa$ & $f_{1}(\mathrm{GHz})$ & $f_{2}(\mathrm{GHz})$ & $\kappa$ \\
\hline$a$ & 15.778 & 17.964 & 0.1291 & 15.747 & 17.971 & 0.1313 \\
\hline $2 a$ & 16.650 & 17.130 & 0.0283 & 16.657 & 17.118 & 0.0273 \\
\hline $3 a$ & 16.822 & 16.948 & 0.0071 & 16.825 & 16.940 & 0.0063 \\
\hline $4 a$ & 16.905 & 16.992 & 0.0015 & 16.867 & 16.894 & 0.0011 \\
\hline
\end{tabular}

the eigenmode of this waveguiding band can be written as a superposition of the individual cavity modes. For $n$ cavities this can be written as

$$
\vec{E}(\vec{r})=E_{0} \sum_{n} e^{-i n k \Lambda} \vec{E}_{\Omega}(\vec{r}-n \Lambda \hat{x})
$$

A simple expression for the dispersion relation of the waveguiding band is given by

$$
\omega(k)=\Omega[1+\kappa \cos (k \Lambda)],
$$

where $\kappa=\left|\beta_{1}-\alpha_{1}\right|$ is the coupling constant parameter, which can be obtained from the splitting of the eigenmodes of two coupled cavities. After obtaining $\Omega, \omega_{1}$, and $\omega_{2}$ from measurements or simulations, one can determine $\alpha_{1}$ and $\beta_{1}$ values by using Eqs. (2a) and (2b). The bandwidth of the guided modes is proportional to the coupling constant and to the single-cavity frequency

$$
\Delta \omega=2 \kappa \Omega \text {. }
$$

Thus, an alternative method for obtaining the coupling constant is measuring the bandwidth of the waveguide and the resonance frequency of an isolated cavity.

Based on this formalism, we investigate the dependence of the coupling constant to the distance between the cavities. Two cavities are formed by removing rods within the PC. The distance between the cavities is changed by an integer multiple of the crystal period from $a$ to $4 a$. By determining the eigenfrequencies from the transmission spectra, the TB coupling constant is calculated. Table I lists the measured and simulated data for the eigenmode frequencies of two coupled cavities and the corresponding calculated TB coupling constant.

In Fig. 3, we plot the calculated coupling constant as a function of the distance between the cavities. Since the spatial extent of the modes decays exponentially, the coupling constant, which essentially depends on the overlap integral of the eigenmodes, also shows an exponential decay. Here, we observe an interesting behavior: when the intercavity distance is $a$, the two cavities actually merge to into a single multimode cavity of size $2 a$, and the modes of this cavity are strongly interacting. Yet, the resulting mode splitting can be described within the TB formalism and the coupling constant fits to the exponential decay behavior very well, as shown in Fig. 3. However, second nearest neighbor couplings become significant with decreasing intercavity distance, therefore, the extension of this particular case to a waveguide (which, actually becomes a straight waveguide) may deviate from TB formalism in its transmission characteristies.

We note that the exponential dependence is obtained between the nondegenerate fundamental mode of the cavities. In the case of modes with nonisotropic spatial extension, the overlap of the fields, and hence the coupling constant as a function of intercavity distance may deviate from the exponential behavior.

\section{THE PHASE SPECTRA OF COUPLED CAVITIES}

The phase information of the transmitted electromagnetic wave is required to determine the dispersion of PC structures. Various experimental studies are performed to determine the band structure of two-dimensional and threedimensional photonic crystals for different frequency regimes. ${ }^{24,25}$ The frequency-dependent phase shift is usually measured by time-domain spectroscopy ${ }^{26-28}$ or using a network analyzer. Robertson et al. ${ }^{26}$ investigated the band structure of alumina based 2D PC's using coherent microwave transient spectroscopy. Kitahara et al. ${ }^{27}$ measured the amplitude and phase shift of transmission spectra of methylpentene-polymer-based photonic crystals by terahertz time-domain spectroscopy, and found out that the phase shift per crystal plane is equal to $\pi$. On the other hand, we have found, to our knowledge, that a direct measurement of the phase shift through CCW's was not reported before, notwith-

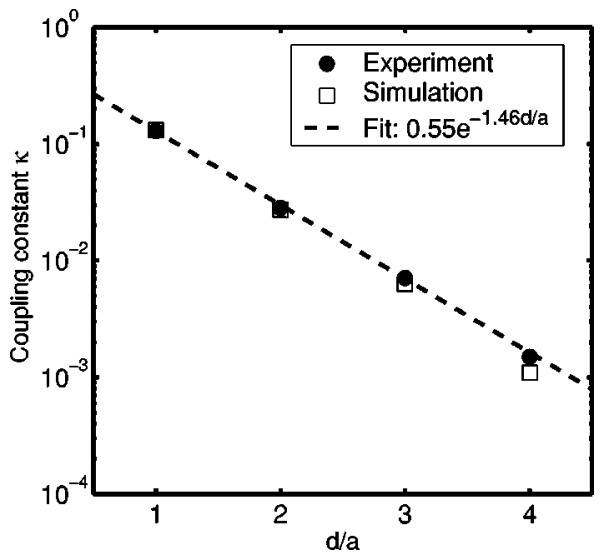

FIG. 3. Measured (solid circles) and simulated (open squares) tight-binding coupling constant as a function of the distance between the cavities. The dashed line denotes the exponential fit. 

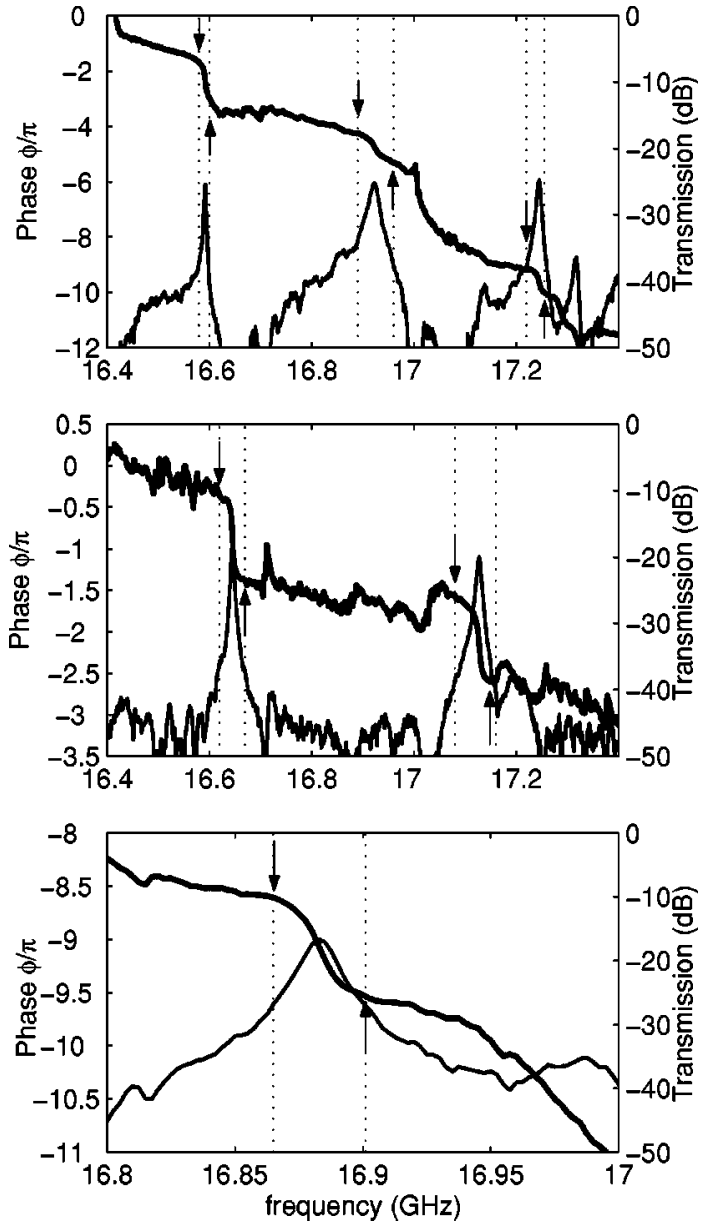

FIG. 4. The phase shift (thick lines) across a single cavity (bottom) and two (middle) and three (top) coupled cavities. Thin lines denote the respective transmission spectrum. Arrows indicate the phase shift through individual cavities.

standing the studies involving CCW's in the context of optical delay lines. In this section of the paper, we are addressing this overlooked issue. As we will present shortly, understanding how the phase advances through a CCW provides a clear and solid basis for interpreting the operation of CCW-based MZI structures.

The phase measurements in our study are performed with an HP 8510C network analyzer. The instrument itself provides the phase of the transmitted signal $\left(S_{12}\right.$ in the $S$-parameter convention), between $[-\pi,+\pi]$, as a function of frequency. These raw data are then "unwrapped" by adding $2 \pi$ at the $\pm \pi$ jumps, to obtain the phase spectra. Since the absolute phase is meaningless, the phase is measured with respect to a calibration. We first perform the calibration in air, by removing the photonic crystal between the antennas, and then measure the relative phase of the relevant structure.

Figure 4 shows the measured phase change through single cavity and two and three coupled cavities along with the corresponding transmission spectra. It is evident that the net phase shift through a cavity, and through each of the coupled cavities, is equal to $\pi$.

The phase shift has its origin in that the cavity in a photonic crystal resembles a Fabry-Perot cavity. The transmis-

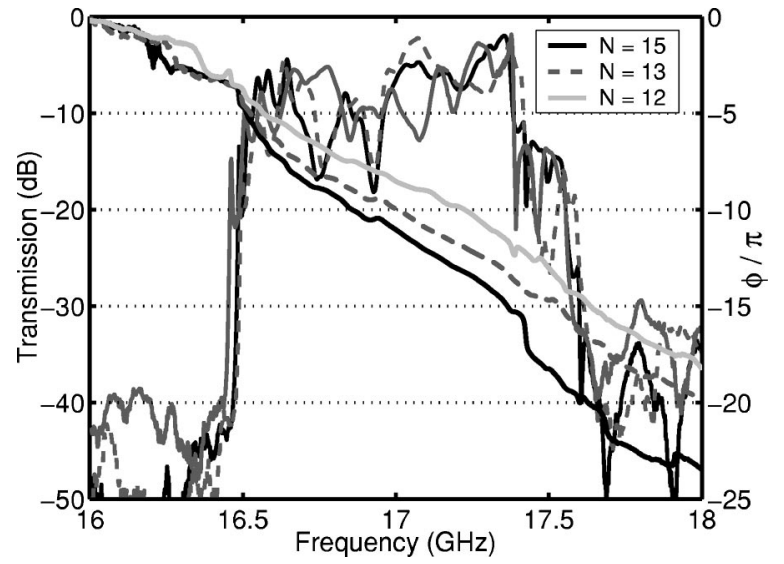

FIG. 5. The phase (lines extending from zero on the upper left) and transmission spectra of CCW's consisting of $N=15$ (solid black), 13 (dashed black), and 12 (solid gray) cavities, respectively.

sion maximum occurs at the frequency corresponding to a standing wave within the cavity. So when the frequency is changed across the resonance, the induced phase shift becomes $\pi$. When many cavities are coupled to form a CCW, each cavity acts as a Fabry-Perot resonator, and the phase difference between the neighboring maxima in the guiding band should be $\pi$. As a result, the total phase shift across the waveguide should become $N \pi, N$ being the number of cavities. In Fig. 5, we show the phase shift across the guiding band of CCW's having 12, 13, and 15 cavities, respectively. The width of the guiding band of all three CCW's are similar. This is expected, since for a sufficiently large number of cavities, the guiding band only depends on the coupling constant and the resonant frequency of a single cavity, as given by Eq. (5). We observe that the phase spectra scale proportional to the number of cavities. The phase at the lowfrequency edge of the guiding band is at $4 \pi$, which then advances to $16 \pi, 17 \pi$, and $19 \pi$ at the high frequency edge of the guiding band. This type of phase shift is also present across the transmission band of the photonic crystals, where

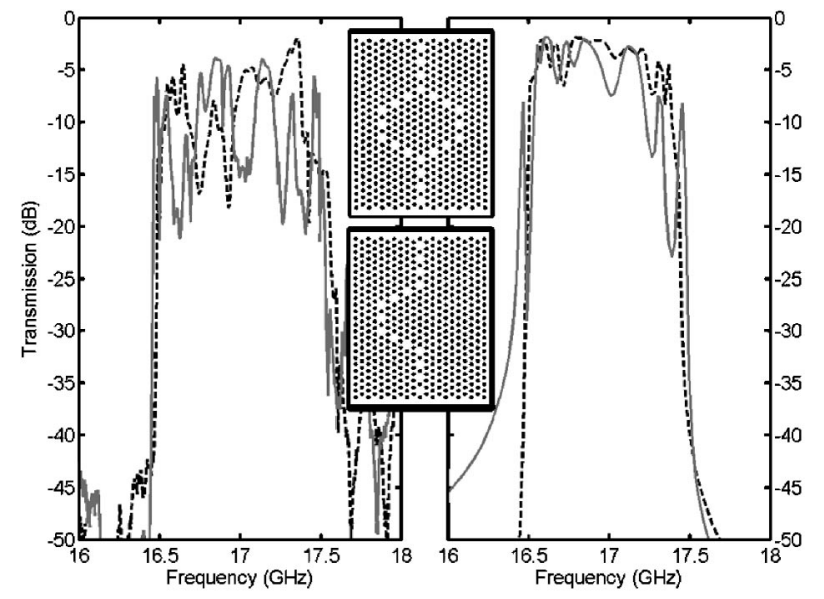

FIG. 6. Comparison of the transmission spectra of a $7 \times 7 \mathrm{MZI}$ (solid lines, top schematic) to one of its isolated branches (dashed lines, bottom schematic). Left panel shows measurements; right panel shows simulations. 

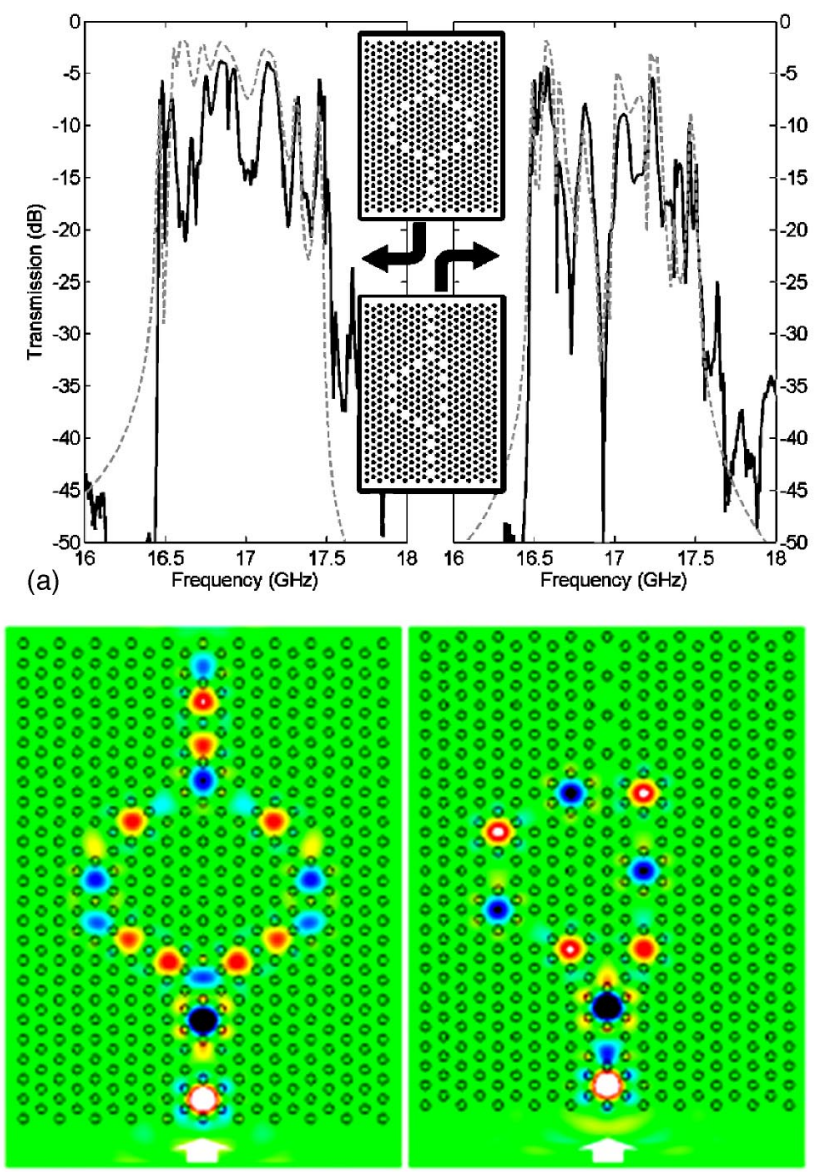

(b)

FIG. 7. (Color online) (a) Measured (solid lines) and simulated (dashed lines) transmission spectra of $7 \times 7$ and $7 \times 5$ MZI structures, depicted in the middle schematics. (b) Simulated electric field profile at $f=16.88 \mathrm{GHz}$ for $7 \times 7$ (left) and $7 \times 5$ (right) MZI, respectively. The arrow at the bottom indicates the input port.

the induced phase shift (i.e., number of transmission maxima) in the frequency region corresponds to the number of crystal planes across the sample in the chosen propagation direction. ${ }^{27,28}$

\section{PHASE SHIFT ANALYSIS OF MACH-ZEHNDER INTERFEROMETER}

In this section, we conduct a phase shift analysis of the CCW-based MZI structures in 2D PC's in view of the results presented in the preceding section. To our knowledge, the operation mechanism of the CCW-based MZI in terms of explicitly measured phase shift differences between the interferometer branches has not been demonstrated before.

We have constructed various MZI structures consisting of two CCW branches connected via $\mathrm{Y}$ junctions to input and output $\mathrm{CCW}$ ports. The input and output ports are along the $\Gamma K$ direction of the $\mathrm{PC}$, each having four cavities. In the following, we denote the MZI structures by the notation $(m \times n)$ according to the number of cavities only in the branches (i.e., the cavities in the input and output ports are not included in the notation). For instance, $7 \times 5$ denotes a MZI with 7 cavities on one branch and 5 on the other.

First, we demonstrate that an $(n \times n)$ symmetric MZI does not exhibit a dip in the transmission spectrum, since the field traversing either branch acquires the same amount of phase shift. Figure 6 compares the transmission spectrum of a 7 $\times 7 \mathrm{MZI}$ to that of an isolated single branch. The measurements on the left panel and simulations on the right panel both show that the double branch and the single branch exhibit very similar transmission characteristics. We note that the flatness of the guiding band obtained in the simulations is not present in the measurements. This is related to the irregularities (small disordering of the rods) of the real PC, which are not present in the simulations. The fluctuations are more pronounced for the double branch, as the interfering fields are more sensitive to small differences in the branches.

In contrast, the transmission spectra of a $7 \times 5$ MZI exhibits a distinct dip at $f=16.88 \mathrm{GHz}$ of about $-60 \mathrm{~dB}$ as shown in Fig. 7. Note also the very good agreement between the simulation and experiment for both the $7 \times 7$ and $7 \times 5$ MZI structures. In the computed field mode at $f$ $=16.88 \mathrm{GHz}$, it is evident that the field interferes destructively at the output $\mathrm{Y}$ junction of the $7 \times 5 \mathrm{MZI}$.

To analyze the dip, we assume that the field at the output $\mathrm{Y}$ junction can be written as a superposition of the field at either branch:

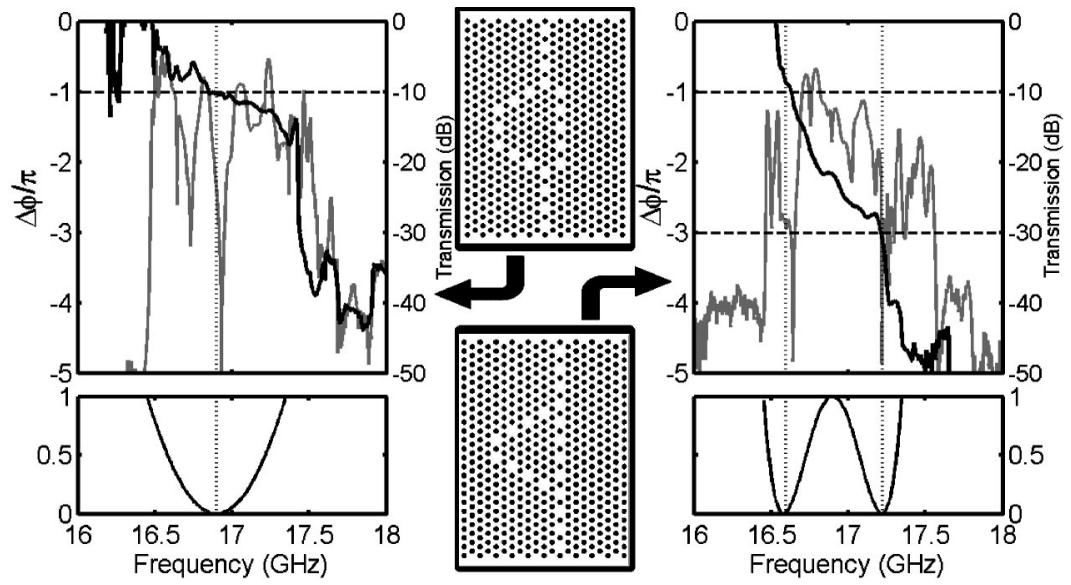

$085108-5$
FIG. 8. Phase (solid black) and transmission (solid gray) spectra of $7 \times 5$ (left) and $8 \times 4$ (right) MZI structures (see middle schematics). The lower figures show the transfer function of Ref. 21. Horizontal dashed lines denote the odd- $\pi$ multiples of the phase difference, whereas vertical dotted lines denote the zeros of the transfer function. 


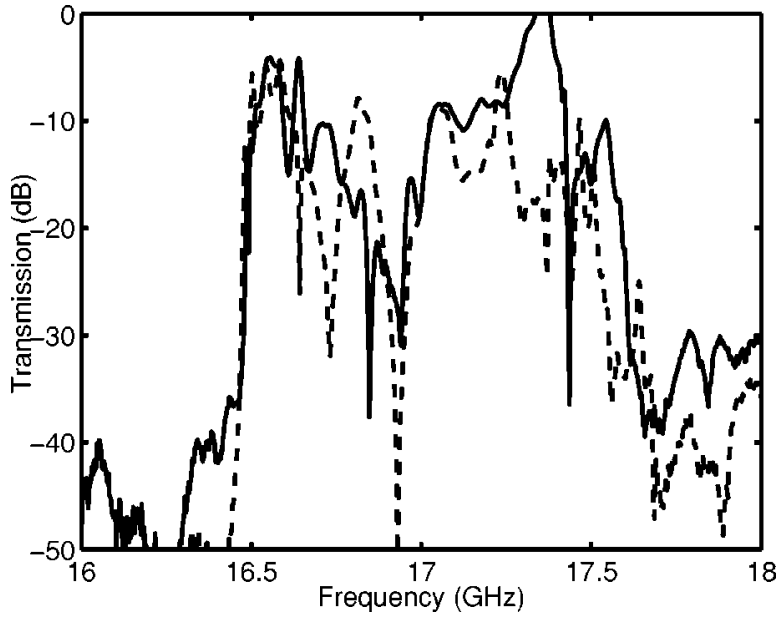

FIG. 9. Comparison of the transmission spectrum measured for full $7 \times 5$ MZI (dashed line) and that of constructed by superposition of the fields of individual isolated branches (solid line).

$$
E_{\text {out }}(f) e^{i \phi_{\text {out }}(f)}=E_{1}(f) e^{i \phi_{1}(f)}+E_{2}(f) e^{i \phi_{2}(f)},
$$

where $E_{1,2}(f)$ and $\phi_{1,2}(f)$ denote the frequency-dependent amplitude and phase of the respective branch field. For an ideal MZI, the amplitude will be independent of the branch. Consequently, the outgoing field vanishes whenever the phase difference between the branch fields $\Delta \phi=\phi_{1}-\phi_{2}$ is an odd multiple of $\pi$ (we postpone the discussion of superposition and equal amplitude assumptions further below). In the preceding section, it is demonstrated that the shift in phase across the guiding band of $N$ coupled cavities is equal to $N \pi$. Since $\Delta N=2$ for the $7 \times 5$ structure, the difference between the phase shifts of the respective branches will be equal to $\pi$ within the guiding band once. Similarly for an $8 \times 4$ structure, where $\Delta N=4$, two interference dips will appear for $\Delta \phi=\pi$ and $\Delta \phi=3 \pi$ as $\Delta \phi$ advances from 0 to $4 \pi$ across the guiding band. Figure 8 demonstrates the matching between the odd $-\pi$ multiples of the phase difference and the transmission dip.

We have also included transfer function calculations according to the simple model of Ref. 20. There, the transfer function of the MZI with input output directional couplers is given by

$$
\begin{aligned}
& T_{11}(f)=\sin ^{2}\left[\frac{\Delta N}{2} \arccos \left(\frac{f-f_{0}}{k f_{0}}\right)\right], \\
& T_{21}(f)=\cos ^{2}\left[\frac{\Delta N}{2} \arccos \left(\frac{f-f_{0}}{k f_{0}}\right)\right],
\end{aligned}
$$

which depends only on the eigenfrequency of an isolated cavity $f_{0}$, coupling constant $\kappa$, and the difference in the num- ber of cavities $\Delta N$. According to this formulation, the transfer function exhibits a dip for $\Delta N=2$ and two dips for $\Delta N$ $=4$ within the guiding band. We observe that both cases are in good agreement with the measured transmission dips and the odd- $\pi$ phase difference positions.

Should we expect a perfect match between the odd- $\pi$ multiples of the phase difference and the transmission dip? We know that the transmission band includes resonance peaks equal to the number of cavities. Therefore, the transmission amplitude at a particular frequency within the guiding band will be intrinsically different on each of the CCW branches, containing a different number of cavities, respectively. Hence, the condition $E_{1}(f)=E_{2}(f)$ may not be satisfied, which, in turn causes a mismatch.

Another issue is the coupling: in Sec. II, the cavity coupling is shown to be significant for separations as large as 4 times the lattice period. Thus, for cavities close to the $\mathrm{Y}$ junctions, both intrabranch and interbranch interactions are present, which renders the superposition idea of isolated branch fields to an approximation for the full MZI structure. To demonstrate this, we compare the transmission spectrum constructed from the superposition of isolated branch fields to that of the full structure for $7 \times 5 \mathrm{MZI}$ :

$$
\log _{10}\left|E_{7 \times 5} e^{i \phi_{7 \times 5}}\right|^{2} \leftrightarrow \log _{10}\left|E_{7} e^{i \phi_{7}}+E_{5} e^{i \phi_{5}}\right|^{2} .
$$

As can be seen in Fig. 9 the superposed field does indeed have a dip around that of full MZI, but the discrepancies throughout the guiding band are evident. Clearly, the details of interbranch interactions depend on the particular interferometer geometry.

\section{CONCLUSION}

In this paper, we have investigated the confinement, coupling properties, and the phase spectra of single- and coupled-cavity structures. The resonance frequency of cavities is found to be independent of the confinement strength. The exponential dependence of the cavity coupling constant is obtained experimentally, which agrees very well with calculated parameters of the tight-binding formalism. We have experimentally demonstrated that the phase shift across the localized mode of a cavity is equal to $\pi$. In a CCW consisting of $N$ number of cavities, the induced phase shift adds up exactly to $N \pi$. This result is utilized to analyze the transmission spectra of CCW-based Mach-Zehnder interferometers. We demonstrate that the phase difference between individual branches by odd multiples of $\pi$ corresponds to transmission dips in the full Mach-Zehnder structure. Deviations from this matching are discussed in terms of the interbranch coupling and of different transmission amplitudes due to the different number of cavities. 
${ }^{1}$ A. Mekis, J. C. Chen, I. Kurland, S. Fan, P. R. Villeneuve, and J. D. Joannopoulos, Phys. Rev. Lett. 77, 3787 (1996).

${ }^{2}$ S. Lin, E. Chow, V. Hietala, P. R. Villeneuve, and J. D. Joannopoulos, Science 282, 274 (1998).

${ }^{3}$ A. Chutinan and S. Noda, Phys. Rev. B 62, 4488 (2000).

${ }^{4}$ A. Chutinan and S. Noda, Appl. Phys. Lett. 75, 3739 (1999).

${ }^{5}$ T. Baba, N. Fukaya, and J. Yonekura, Electron. Lett. 35, 654 (1999).

${ }^{6}$ M. Tokushima, H. Kosaka, A. Tomita, and H. Yamada, Appl. Phys. Lett. 76, 952 (2000).

${ }^{7}$ C. J. M. Smith, H. Benisty, S. Oliver, M. Rattier, C. Weisbuch, T. F. Krauss, R. M. De La Rue, R. Houdré, and U. Oesterle, Appl. Phys. Lett. 77, 2813 (2000).

${ }^{8}$ J. Moosburger, M. Kamp, A. Forchel, S. Olivier, H. Benisty, C. Weisbuch, and U. Oesterle, Appl. Phys. Lett. 79, 3579 (2001).

${ }^{9}$ A. Chutinan, M. Okano, and S. Noda, Appl. Phys. Lett. 80, 1698 (2002).

${ }^{10}$ A. Talneau, L. L. Gouezigou, N. Bouadma, M. Kafesaki, C. M. Soukoulis, and M. Agio, Appl. Phys. Lett. 80, 547 (2002).

${ }^{11}$ A. Yariv, Y. Xu, R. K. Lee, and A. Scherer, Opt. Lett. 24, 711 (1999)

${ }^{12}$ M. Bayindir, B. Temelkuran, and E. Ozbay, Phys. Rev. Lett. 84, 2140 (2000); Appl. Phys. Lett. 77, 3902 (2000).

${ }^{13}$ M. Bayindir, E. Ozbay, B. Temelkuran, M. M. Sigalas, C. M. Soukoulis, R. Biswas, and K. M. Ho, Phys. Rev. B 63, 081107 (2001)

${ }^{14}$ E. Lidorikis, M. M. Sigalas, E. N. Economou, and C. M. Soukoulis, Phys. Rev. Lett. 81, 1405 (1998).
${ }^{15}$ Y. Sugimoto, N. Ikeda, N. Carlsson, K. Asakawa, N. Kawai, and K. Inoue, Appl. Phys. Lett. 79, 4286 (2001).

${ }^{16}$ M. Loncar, D. Nedelkovic, T. Doll, J. Vuckovic, A. Scherer, and T. P. Pearsall, Appl. Phys. Lett. 77, 1937 (2000).

${ }^{17}$ S. Lan, S. Nishikawa, H. Ishikawa, and O. Wada, J. Appl. Phys. 90, 4321 (2001).

${ }^{18}$ S. Lan, S. Nishikawa, Y. Sugimoto, N. Ikeda, K. Asakawa, and H. Ishikawa, Phys. Rev. B 65, 165208 (2002).

${ }^{19}$ M. Soljačić, S. G. Johnson, S. Fan, M. Ibanescu, E. Ippen, and J. D. Joannopoulos, J. Opt. Soc. Am. B 19, 2052 (2002).

${ }^{20}$ A. Martinez, A. Griol, P. Sanchis, and J. Marti, Opt. Lett. 28, 405 (2003).

${ }^{21}$ E. Yablonovitch, Phys. Rev. Lett. 58, 2059 (1987).

${ }^{22}$ N. Stefanou and A. Modinos, Phys. Rev. B 57, 12127 (1998).

${ }^{23}$ A. Martinez, A. Garcia, P. Sanchis, and J. Marti, J. Opt. Soc. Am. A 20, 147 (2003).

${ }^{24}$ S. L. McCall, P. M. Platzman, R. Dalichaouch, D. Smith, and S. Schultz, Phys. Rev. Lett. 67, 2017 (1991).

${ }^{25}$ E. Yablonovitch, T. J. Gmitter, and K. M. Leung, Phys. Rev. Lett. 67, 2295 (1991).

${ }^{26}$ W. M. Robertson, G. Arjavalingam, R. D. Meade, K. D. Brommer, A. M. Rappe, and J. D. Joannopoulos, Phys. Rev. Lett. 68, 2023 (1992); J. Opt. Soc. Am. B 10, 322 (1993).

${ }^{27}$ H. Kitahara, N. Tsumura, H. Kondo, M. W. Takeda, J. W. Haus, Z. Yuan, N. Kawai, K. Sakoda, and K. Inoue, Phys. Rev. B 64, 045202 (2001).

${ }^{28}$ T. Aoki, M. W. Takeda, J. W. Haus, Z. Yuan, M. Tani, K. Sakai, N. Kawai, and K. Inoue, Phys. Rev. B 64, 045106 (2001). 